\section{Lehre zur Technikbewertung in den Lebenswissenschaften}

\author{
von Volker Beusmann und Regine Kollek, \\ Universität Hamburg
}

Moderne Biotechnologien wurden von Beginn ihrer Entwicklung an von gesellschaftlichen Kontroversen begleitet. Vor diesem Hintergrund wurde an der Universität Hamburg ab 1993 der „Forschungsschwerpunkt Biotechnik, Gesellschaft und Umwelt“" (FSP BIOGUM) mit zwei Forschungsgruppen (FG) parallel zu neuen molekularbiologischen Instituten etabliert. $\mathrm{Zu}$ den Aufgaben des FSP BIOGUM gehört neben der Forschung und der Beratung von Politik und Öffentlichkeit auch die Lehre. Die FG Landwirtschaft wird seit Mitte 1993 von Volker Beusmann geleitet, die FG Medizin seit Ende 1995 von Regine Kollek. Dieser Beitrag gibt erstens einen Überblick über die Ausbildungsangebote, zweitens werden Konzepte und Lehrinhalte präsentiert. In einem dritten Teil werden die Aktivitäten des FSP BIOGUM im Hinblick auf die vier vorgegebenen Verbindungslinien zwischen Technology Assessment (TA) und Bildung reflektiert.

\section{Einleitung}

Eine Grundidee bei der Einrichtung des FSP BIOGUM und bei der Formulierung seiner Aufgaben, speziell der Lehraufgaben war es, dass Naturwissenschaftler und Technikentwickler sich im Studium mit den gesellschaftlichen Kontroversen um die Gentechnik in Medizin, Neurowissenschaften, Landwirtschaft und Ernährung auseinandersetzen. Dies war nicht nur ein Anliegen der Bürgerschaft der Freien und Hansestadt Hamburg und des Akademischen Senats der Universität Hamburg, sondern auch von Vertretern der Naturwissenschaften. Das Spektrum der Erwartungen an die TA und die Rolle ihrer Vertreter war allerdings breit gestreut: vom „Genpolizist und Aufpasser“ über den „Gemeinschaftskundelehrer an der Universität" bis zum „Fitnesstrainer für Gentechnikabsolventen zur Abwehr gesellschaftlicher Kritik“. Unser eigenes Selbstverständnis lässt sich hingegen wie folgt umreißen: Wir möchten den Studierenden Angebote machen, die sie zur Selbstentfaltung in Verantwortung für die gesellschaftliche und natürliche Mitwelt nutzen können. Dahinter steht die Hoffnung, nicht nur einen Beitrag zu ihrer Ausbildung und Bildung zu leisten, sondern sie auch darin zu fördern oder sie dafür zu gewinnen, sich den großen Zukunftsfragen der Menschheit in ihrem „Teil der Welt" zu stellen. ${ }^{1}$ Ihre Persönlichkeit bilden können sie nur selbst (Dörpinghaus 2009, S. 5).

\section{Bildungsangebote FSP BIOGUM im Überblick}

Im Kern der Lehre stehen Pflichtveranstaltungen der beiden Autoren dieses Artikels bei den Naturwissenschaftlern und Technikentwicklern in der Biochemie / Molekularbiologie, Botanik und zwischenzeitlich der Bioinformatik, auf die weiter unten (siehe Abschnitt 3) ausführlicher eingegangen wird. Daneben werden weitere Lehrveranstaltungen auch von Mitarbeitern oder gemeinsam mit ihnen angeboten, die hier im Überblick dargestellt werden. ${ }^{2}$ Inhaltlich erstrecken sich die Lehrveranstaltungen auf die modernen Biotechnologien inkl. Gentechnik in Landwirtschaft und Ernährung sowie in Medizin und Neurowissenschaften, d. h. sowohl die sog. Grüne als auch die Rote Gentechnik. Die „Weiße“ Biotechnologie, die an der TU Harburg als Teil der Ausbildung von Biochemikern / Molekularbiologen (jetzt Molecular Life Sciences) gelehrt wird, ist nicht Thema der vom FSP angebotenen Lehre, da sie in der Gesellschaft weniger kontrovers ist.

Neben der Pflichtlehre für Studierende gibt es in den beiden Forschungsgruppen Lehrveranstaltungen für den Wahl- und Wahlpflichtbereich. Dazu gehören Seminare, die mit Doktoranden und Mitarbeitern durchgeführt werden und in denen über laufende Arbeiten und gemeinsam interessierende Themen diskutiert wird - teils auch mit externen Referierenden. Die Mitarbeiter beider Forschungsgruppen bieten ferner Lehrveranstaltungen als Privatdozenten oder im Rahmen akademischer Qualifizierungen in politik- und sozialwissenschaftlichen Disziplinen sowie an anderen Hochschulen an.

An Studierende aller Fakultäten und die allgemeine Öffentlichkeit richten sich Ringvorlesungen, die teils mit Mitarbeitern eigenständig, teils in Kooperation mit Vertretern anderer Forschungsgruppen organisiert wurden. Zum 
Teil wurden parallel zu den Vorlesungen vertiefende Seminare angeboten. Besonders interessant - gerade auch im Hinblick auf eigenes Lernen - finden wir Co-Teaching-Seminare, z. B. zur Biodiversität aus Sicht von Ökologen, Ökonomen und Politikwissenschaftlern, die besonders attraktiv sind für Fortgeschrittene und Mitarbeiter.

Ein fester Bestandteil des Lehrangebots aus dem FSP BIOGUM ist weiterhin ein interdisziplinäres Ethikseminar, das seit vielen Jahren in jedem Semester von Mitgliedern der FG Medizin gemeinsam mit Angehörigen der medizinischen Fakultät organisiert und im Fachbereich Medizin für Hörer aller Fakultäten und die Öffentlichkeit angeboten wird. In diesem Rahmen wird unter regelmäßiger Beteiligung externer Referenten ein breites Spektrum von Themen diskutiert, die von speziellen medizinethischen Problemen bis hin zu Fragen der Gesundheitspolitik und der gesundheitsrelevanten kulturellen Entwicklung reichen. Gemeinsam mit Kollegen aus der Medizin wird darüber hinaus für Studierende der Medizin jeweils im Sommersemester ein Wahlfach „Geschichte, Ethik, Technikfolgenabschätzung und Theorie der Medizin“" angeboten.

Ein Schwerpunkt des Bildungsangebots der FG Landwirtschaft liegt auf Veranstaltungen mit Lehrern und Schülern. Einzelveranstaltungen werden für Schulklassen parallel zu Vorlesungen und Führungen von Naturwissenschaftlern des Biozentrums angeboten. Mit dem Fachbereich Erziehungswissenschaften und dem Landesinstitut für Lehrerbildung und Schulentwicklung wurde in einem Seminar für Biologie-, Philosophie- und Religionslehrer zur Unterstützung für fachübergreifende Unterrichtseinheiten kooperiert. Unter zusätzlicher Einbindung der TUTech Harburg wurde schließlich der Online-Diskurs „BioTalk“ mit Hamburger Schülern verschiedener Schultypen durchgeführt, die Materialien stehen über den Hamburger Bildungsserver zur Verfügung. ${ }^{3}$

\section{Konzepte und Inhalte der Pflichtlehre}

Gemeinsames Ziel der Lehrveranstaltungen ist es, die Studierenden zur Reflexion über ihr Selbst-, Gesellschafts- und Naturverständnis anzuregen und in beiden Themenbereichen eine Einführung in die Problemfelder, Konzepte,
Theorien und Methoden sowie Resultate der TA zu geben.

\subsection{Technikfolgenabschätzung zur Gen- und Biotechnik in der Landwirtschaft}

In der TA zur Grünen Gentechnik (Volker Beusmann und Mitarbeiter) werden die großen Herausforderungen der Menschheit mit der Club-of-Rome-Studie „Grenzen des Wachstums" von 1972, der Rio-Konvention zur Nachhaltigen Entwicklung und den seither international verfolgten Aktivitäten eingeführt, um den Studierenden nahe zu bringen, welche Aufgaben sich ihnen in Zukunft stellen. Danach erfolgt ein Abriss über die Hintergründe der Entstehung der TA sowie eine Gegenüberstellung von TAIdeal-Vorstellungen und ihren realen Grenzen. Diese Gegenüberstellung hat drei Ziele:

- zu sensibilisieren, dass TA nicht in ihrem Leistungsvermögen überfordert oder überschätzt wird;

- aufzuzeigen, mit welchen Methoden sie auf die Grenzen reagiert und wie mit der prinzipiellen Unsicherheit und Offenheit der $\mathrm{Zu}$ kunft umgegangen wird;

- einen weiten Rahmen abzustecken, in dem z. B. Arbeiten Einzelner für universitäre Qualifikationen verortet und ihre Resultate angemessen interpretiert werden können.

Die TA-Einführung endet mit der reflexiven Wendung in der Betrachtung von TA als Wissenschaft über Wissenschaft und Technik mit ihren ambivalenten Rollen als Problemlöser und -verursacher, teils in der zeitlichen Konstellation eines „Problemverlagerers“.

Diese TA-Konzeption baut auf der VDIRichtlinie Technikbewertung (1991) auf, deren Elemente von der Darstellung der Technik und ihrer Potenziale bis zur Vorbereitung gesellschaftlich legitimierter Entscheidungen als Stufen für die Lehrveranstaltung gewählt werden, wobei auf einige Schwerpunkte und Erweiterungen besonderer Wert gelegt wird (ausführlicher in Beusmann 2007): Neben der Analyse, Bewertung und Gestaltung der Chancen und Risiken von Technologien besonders betont wird die Rolle von Alternativen sowie von Verhaltensweisen und Institutionen, verstanden als die informellen bis formalen Spielregeln menschlichen Zusammenlebens. Dabei werden 
zwei Blickwinkel in Beziehung gesetzt: zum einen der der Komplementarität, der Einbettung von Technologien in Verhaltensweisen und Spielregeln, zum anderen der der Konkurrenz als Lösungsstrategien. Man kann den Hunger auf der Welt mit besseren Technologien vermindern (z. B. Saatgut, Anbausysteme, Logistik und Verarbeitung), mit veränderten Verhaltensweisen (z. B. Reduzierung des Fleischkonsums in Industrieländern) oder mit veränderten Spielregeln (z. B. verbesserter Bildung insbes. für Frauen, Zugang zu Mikrokrediten, Marktzugang, fairer Handel). Entsprechend vielfältig, differenziert und gut abgestimmt sollten die Strategien zur Hungerreduzierung sowie die Bildungs-, Forschungs- und Entwicklungsinvestitionen ausfallen. Die Studierenden werden mit folgenden Fragen konfrontiert: Wo versprechen sie sich komparative Vorteile für die von ihnen verfolgten Optionen? Welche Voraussetzungen und Abstimmungen mit Verhaltensweisen und Spielregeln sind für die Umsetzung erforderlich? Insgesamt steht diese TA-Konzeption der Nachhaltigkeitsforschung sehr nahe.

Der nächste Themenblock ist eine Einführung in die Kosten-Nutzen-Theorie und die Ökonomie technischen und strukturellen Wandels. Da er für das Verständnis der globalen Entwicklung moderner Biotechnologie wesentlich und für Naturwissenschaftler fachfremd ist, wird er einführend als Vorlesung präsentiert. Danach folgen - in Seminaren auch als Referate - Themen über den Stand und die Verbreitung von gentechnisch veränderten Pflanzen (gvP) sowie unterschiedliche Visionen für die von Landnutzung und Ernährung; damit wird der Streit um die Gentechnik in den Rahmen der Konflikte um die Gestaltung der Zukunft gestellt (food, feed, fiber, fuel; high tech vegetarian, personalisierte Ernährung, funktionelle Lebensmittel aus gvP, Ökologischer Landbau). Es schließen sich Analysen zu den Folgen an: Ökonomische Folgen von gvP werden exemplarisch für Landwirte, Verbraucher und für die Konzentrationsprozesse in Züchtung, Verarbeitung und Handel untersucht. Ökologische Implikationen werden entweder in einer allgemeinen Form als Konzeptrahmen dargestellt oder mit spezifischen Fragestellungen als Fallstudien behandelt und ggf. von Mitarbeiterinnen betreut. Das Gleiche gilt für die allgemeinen Prüfverfahren für gesundheitliche Implikationen, spezifische Fall- studien sowie Regulierungsfragen. Zur partizipativen TA als Ansatz des friedlichen Umgangs mit Konflikten werden Grundlagen und ausgewählte Fallstudien erörtert. Die Entwicklung von Weltbevölkerung und Welternährung gehören in der Regel in die Seminarprogramme, „Golden Rice“" wird häufig als eine Fallstudie gewählt. Abschließend werden Gerechtigkeitsvorstellungen, die Beziehungen zwischen TA und Ethik sowie die gesetzliche Regulation im Vergleich USA und Europa behandelt.

Für Seminare gibt es eine Reihe von weiteren Themen, die zur Wahl gestellt werden. Hierzu gehören die Darstellung der Gentechnik in den Medien und Akzeptanzstudien, methodische Themen (wie Delphimethode und Szenariotechnik), Risikowahrnehmung, -abschätzung, -management, -kommunikation und -politik, aber auch Themen wie „Patentrecht vs. Sortenschutz", Haftungsrecht, Koexistenz von Landbauformen mit und ohne Gentechnik, Biodiversität (zwischen Bioprospektierung und Biopiraterie) und Biowaffen. Daneben wird auch aus eigenen Forschungen berichtet.

\subsection{Technikfolgenabschätzung zur Gen- und Biotechnik in Medizin und Neu- rowissenschaften}

Die TA im Bereich der Medizin und Neurowissenschaften (Regine Kollek und Mitarbeiter) versucht, die komplexen Implikationen biomedizinischer Technikentwicklung zu erfassen, die von unmittelbaren medizinischen und psychischen Auswirkungen solcher Technologien auf das Individuum bis hin $\mathrm{zu}$ ethischen und gesellschaftlichen Folgen reichen. Damit steht sie zwischen der in der Medizin seit Längerem etablierten „Evidenz-basierten Medizin“ (EbM) auf der einen Seite, die vorliegende Erkenntnisse zu den unmittelbaren Auswirkungen medizinischer Techniken auf die Patienten systematisch zu bewerten versucht, und der medizinischen Ethik andererseits, die die normativen Rahmenbedingungen für die Anwendung der entsprechenden Entwicklungen klären will. TA im Bereich biomedizinischer Techniken und Entwicklungen versucht, auf diese heterogenen Herausforderungen zu reagieren und die Implikationen neuer biomedizinischer Techniken nicht nur für Individuen, sondern auch für Gesundheitssystem und Gesellschaft zu klären. 
Dieser umfassende Anspruch wirft methodische und konzeptionelle Fragen auf, auf die die FG mit der Entwicklung eines eigenen Konzepts zum „BioMedical Technology Assessment" reagiert hat. ${ }^{4}$ Grundsätzlich geht es dabei darum, der Multidimensionalität möglicher Technikimplikationen sowie der Multiperspektivität ihrer Bewertung gerecht zu werden.

Dieses Konzept, das eine Weiterentwicklung existierender Health-Technology-Assessment-Ansätze darstellt, spiegelt sich auch im Aufbau der Lehre wider. Als Problemeinstieg dienen hier zumeist aktuelle, sich medial widerspiegelnde Kontroversen im Bereich der biomedizinischen Forschung und Entwicklung. Beispiele für die sich daraus auf verschiedenen Ebenen ergebenden Herausforderungen sind die Stammzellforschung oder die Forschung zum Neuroenhancement. Anknüpfend an bzw. in Ergänzung zu den TA-Konzepten im Bereich der Grünen Gentechnik werden dann die verschiedenen, für Medizin und Neurowissenschaften relevanten TA-Ansätze und Konzepte vorgestellt: Methodik und Systematik klinischer Studien, Metaanalysen solcher Studien im Rahmen der EbM, verschiedene Konzepte der gesundheitsökonomischen Evaluation, soziale Effekte biomedizinischer Technologien bis hin zu gesellschaftlichen Implikationen und ethischen Fragen. Orientierungsrahmen ist dabei das Gesamtkonzept des Health- bzw. BioMedical-Technology-Assessment, in dem neben den naturwissenschaftlich erfassbaren Effekten auch ökonomische, psychische, soziale, rechtliche, kulturelle und ethische Implikationen berücksichtigt werden.

Die Vorstellung der verschiedenen TAAnsätze und Vorgehensweisen erfolgt exemplarisch anhand von Fallbeispielen, die in Abhängigkeit von Studiengang, aktuellen Problemlagen oder relevanten eigenen Forschungsprojekten wechseln können. ${ }^{5} \mathrm{Zu}$ den behandelten Themenfeldern gehören u. a. Gentests und genetische Screenings, ethische und rechtliche Fragen im Umgang mit genetischen Daten, Biobanken, Stammzellforschung,medikamentöse Leistungssteigerung, Verschiebung des Gesundheits- / Krankheitsbegriffs durch diagnostische und therapeutische Möglichkeiten, Formen der sozialen Anerkennung bzw. Ausgrenzung durch biomedizinische und neurowissenschaftliche Entwicklungen, Implikationen der Reprodukti- onsmedizin aus Sicht der verschiedenen Beteiligten, aber auch eine kritische Reflexion formaler Bewertungsindikatoren wie das der ,quality adjusted life years" (QALY). Den Abschluss bildet zumeist eine Diskussion des Verhältnisses von Technik und Gesellschaft. In den Seminaren befassen sich die Studierenden vertiefend mit Teilaspekten dieser Themen oder greifen andere auf, die in der Vorlesung nicht oder nur oberflächlich behandelt werden konnten.

Ziel dabei ist, den Studierenden einen Überblick über die Regelhaftigkeiten und Unsicherheiten der Technikentwicklung im Bereich von Medizin und Neurowissenschaften, und damit auch über die Möglichkeiten und Grenzen der einschlägigen TA und ihrer Konzepte zu vermitteln. Darüber hinaus sollen die Studierenden für die Perspektivenabhängigkeit von Bewertungen sensibilisiert werden: Es macht einen Unterschied, ob beispielsweise ein Verfahren der künstlichen Befruchtung aus medizinischer oder aus einer bestimmten ethischen Perspektive bewertet wird und ob dies aus der Position von Ärzten, betroffenen Paaren, Gesundheitsökonomen oder Juristen geschieht. Die Bedeutung dieser Perspektivenabhängigkeit von Bewertungspositionen $\mathrm{zu}$ verdeutlichen und aufzuzeigen, wie methodisch zwischen ihnen vermittelt werden kann, ist ein weiteres Ziel, das hier in der Lehre angestrebt wird. Grundsätzlich wird dabei in Vorlesungen versucht, deren monologischen Charakter durch Diskussionsteile aufzulockern und die Studierenden in die argumentative Evaluation spezifischer Fragen einzubeziehen. Dies kann Seminare oder andere, partizipative Formen des Wissenserwerbs nicht ersetzen, vermag aber trotzdem einen konkreten Eindruck von der Komplexität und Multiperspektivität der Folgenabschätzung und -bewertung zu vermitteln, die in allen gesellschaftlichen Bereichen vorfindbar ist.

Generell ist es uns im Rahmen der Lehre zur TA wichtig aufzuzeigen, auf welche Weise die neuen biomedizinischen Entwicklungen die bestehende soziale Ordnung herausfordern ${ }^{6}$ und welche ethischen, rechtlichen und politischen Strategien entwickelt werden, um die dadurch entstehenden neuen gesellschaftlichen Konfigurationen $\mathrm{zu}$ stabilisieren. Angesprochen sind damit „Humantechniken“ wie die Bioethik, das Recht oder die Governance (Nowotny, Testa 2009), deren Bedeutung für eine 
pluralistische, sozial und zukunftsverträgliche Technikentwicklung und Regulierung nicht genug betont werden kann. Die gegenseitige Abhängigkeit von wissenschaftlich-technischer und gesellschaftlicher Entwicklung aufzuzeigen, ist ein Grundanliegen aller Bemühungen zur Vermittlung von TA im Bereich der Lehre.

\section{TA und Bildung im Rückblick und Ausblick}

\subsection{Regeneration der TA-Community}

In Bezug auf die Konsolidierung und den weiteren Ausbau der TA-Community durch die Lehre im Bereich der TA verstehen wir den Begriff der Community in zweifachem Sinn: Im engen Sinn ist damit die (Aus-)Bildung für den TA-Nachwuchs in Universität und Forschung gemeint, im weiten Sinne die (Aus-) Bildung für (natur-)wissenschaftliche Praktiker, die TA in der einen oder anderen Weise im Berufsfeld umsetzen. Bei Letzteren handelt es sich um die bei weitem größere Zahl von Absolventen, die als Multiplikatoren für die Ausbreitung der TA-Ideen in der Gesellschaft und als Kooperationspartner für die transdisziplinäre TA-Forschung von großer Bedeutung sind.

Bisher gibt es keine eigenen Ordnungen für die Promotion oder Habilitation und auch keine Juniorprofessuren in der TA im FSP BIOGUM. Die formale Qualifizierung des TA-Nachwuchses erfolgt in der Regel in der Disziplin, in der der Studienabschluss vorliegt und in Kooperation mit Vertretern dieser Disziplinen. Eigene Promotionsrechte bestehen in der naturwissenschaftlichen (Beusmann) und der medizinischen Fakultät (Kollek). Diese Situation ist insofern unbefriedigend, als dass die Erstgutachter der am FSP BIOGUM unter der Anleitung der Promotionsberechtigten durchgeführten, zumeist interdisziplinären Promotionen oft $\mathrm{zu}$ anderen Fakultäten oder gar Universitäten gehören. Andererseits konnten durch dieses Arrangement Kooperationen mit Personen und Einrichtungen in natur-, sozial- und geisteswissenschaftlichen Fakultäten etabliert werden (u. a. im Rahmen eines von Regine Kollek gemeinsam mit Alfons Bora (Universität Bielefeld) geleiteten und von der Evangelischen Studienstiftung Villigst geförderten Promotionsschwerpunktes), die zur Regeneration der TA-Community und ihrer
Vernetzung beitragen. Da sich bisher für alle Fälle von Qualifikationsarbeiten eine institutionelle Lösung gefunden hat, planen wir derzeit keine Aktivitäten zur Etablierung formaler Ordnungen für reine TA-Abschlüsse.

In der Zielrichtung Universitätslaufbahn wurde die Habilitation von Stephan Albrecht erfolgreich abgeschlossen, eine andere Arbeit liegt der entsprechenden Fakultät vor. Der ehemalige Leiter einer Nachwuchsgruppe, Peter Feindt, ist inzwischen als Senior Lecturer für „City and Regional Planning“ an der Cardiff University tätig.

\subsection{Theorieentwicklung von TA: Impulse aus der Lehre}

Da eine einheitliche theoretische Grundlage für TA bislang kaum definiert werden kann oder als Metatheorie für eine fachbezogene Lehre sehr abstrakt bleibt, beziehen wir uns in der Lehre auf eine Vielzahl von Theorien, Konzepten und Methoden, die zumeist jedoch weder exklusiv für TA entwickelt wurden noch für sie eingesetzt werden. Die Frage ist, ob sich aus der Lehre Impulse ergeben, die sich in theoretischen Ansätzen der TA niederschlagen sollten.

Grundsätzlich besteht bei vielen Studierenden der Naturwissenschaften und der Medizin ein explizites Bedürfnis nach gemeinsamen Problemdiskussionen; beide Gruppen werden im Verlauf ihres Studiums in Universität und Gesellschaft mit Disziplinen überschreitenden Problemlagen konfrontiert, haben aber außerhalb von TA-Lehrveranstaltungen nur wenig institutionalisierte Möglichkeiten, ihre Argumente zu erproben und weiterzuentwickeln.

Dieses Bedürfnis steht in einem gewissen Widerspruch zu dem Bemühen, den Studierenden solides systematisches Wissen zur TA, ihren Konzepten und Methoden zu vermitteln. Die Herausforderung, die sich daraus für die Lehre ergibt - möglicherweise aber auch für die TA als Ganzes - lautet: Wie könnten systematische, theorieorientierte Vorgehensweisen und diskursive Elemente miteinander verknüpft werden? Frühere Lehrexperimente zeigten, dass interaktive und diskursive Formen der Vermittlung auf große Resonanz stoßen. Beispielsweise haben wir in einem Semester auf Anstoß von Studierenden, die Erfahrung in Moderation sowie der Leitung von Jugendgruppen mitbrachten, ein 
ungewöhnliches Experiment durchgeführt: die Simulation einer Konsensus-Konferenz zu einer medizinischen Thematik in Form eines Rollenspiels. Die Mitautorin dieses Beitrags fungierte als fachliche Beraterin im Sachthema, der andere als Verfahrensberater sowie Freunde der Studierenden (aus anderen Disziplinen) als Bürgerjury. Die Mehrzahl der teilnehmenden Studierenden war außergewöhnlich engagiert. Es gab aber auch Umsetzungsprobleme und in einem Fall ging das Rollenspiel schief, weil ein Teilnehmer dem Rollenspiel ablehnend gegenüber stand und er seine eigene Position zu der Rolle, die er übernommen hatte, nicht hinreichend geklärt hatte. Insgesamt war der Lerneffekt für alle Teilnehmer (inklusive Lehrende) jedoch erheblich und überwog den eines Standardseminars. Das mag einerseits daran liegen, dass die Voraussetzungen günstig und das Engagement der Studierenden intensiv waren. Weiterhin stellen solche Lehrformen hohe Ansprüche an das Zeitbudget des Lehrpersonals, was die Etablierung solcher Lehrformen für größere Zahlen von Studierenden schwierig macht. In Anbetracht der Umstrukturierungen des Studiums und auch angesichts der zunehmenden Vorbildung von Schülern im selbständigen und Gruppenlernen planen wir dennoch, konventionelle Formen der Vermittlung von TA-Inhalten (Vorlesungen oder Seminare) verstärkt durch solche unkonventionellen Formen zu ergänzen oder zu ersetzen.

Grundsätzlich gilt es, der doppelten Anforderung - Vermittlung systematischen Wissens und Einbeziehung diskursiver Elemente - in der Lehre gerecht $\mathrm{zu}$ werden. Universitäre TALehre muss sowohl der empirischen Tatsache Rechnung tragen, dass Beteiligung im weitesten Sinne eine grundlegende Voraussetzung für Lernbereitschaft und letztlich auch für die Akzeptanz des Faches ist, als auch der wissenschaftlichen Anforderung genügen, theoretisches Wissen und Kenntnisse über systematische Vorgehensweisen zu vermitteln. Da die uns dafür zur Verfügung stehende Zeit begrenzt ist, kann beides notwendiger Weise nur exemplarisch geschehen. Dies muss dem Erfolg eines solchen Konzepts jedoch nicht abträglich sein.

\subsection{TA-bezogene Kompetenzen und Public Understanding of Science}

Das Spektrum und die Schwerpunkte unserer Aktivitäten sind oben dargelegt. Bisher waren unser TA-Pflichtveranstaltungen auf wenige Studiengänge beschränkt, aber offen für andere - insbesondere Lehramtskandidaten und Gasthörer. Die Herausgeber dieses Schwerpunktheftes fordern eine möglichst breite Vermittlung von TA-Kompetenzen (siehe dazu den Einführungsbeitrag von Beecroft, Dusseldorp und Moniz in diesem Heft). Ähnlich argumentiert der Deutsche Hochschulverband (2009) in der Begründung seiner Forderung „Kein Studium mehr ohne Wissenschaftsgeschichte und Ethik“. Im Gegensatz dazu wird jedoch in unserem Umfeld mit dem Übergang auf die neuen Studiengänge die TA als Pflichtlehre reduziert und in den Studiengängen der „Molecular Life Sciences" die Studierendenzahl verdoppelt oder gar verdreifacht, weshalb wir die TAVeranstaltung deshalb jetzt als Vorlesung mit Abschlussklausur anbieten. Zugleich öffnen sich Zeitfenster für Wahlfächer; in diesen werden aber z. T. überwiegend weitere disziplinäre Spezialisierungen angeboten (z. B. Bioinformatik). Demzufolge laufen die Überlegungen bei uns in folgende Richtungen: Zum einen könnte das Angebot von Mischformen, also Veranstaltungen mit Vorlesungs- und Diskussionsteilen erhöht werden. Zum anderen wäre an eine Einführungsvorlesung in Form eines Co-TeachingAngebots für eine größere Hörerzahl aus unterschiedlichen Fächern mit begleitenden Seminarangeboten für kleinere Gruppen zu denken. Auch über interessierte Studierende, also solche, die über Wahlfächer oder auf andere Weise noch zu Umwegen bereit sind (Winter 2009), wollen wir versuchen, das Interesse an TA zu wecken und die Ausbreitung von TAIdeen zu fördern.

\section{Anmerkungen}

1) Siehe dazu Silbereisen 1997 und Peccei 1979.

2) Siehe im Einzelnen dazu http://www.biogum.unihamburg.de, dort insbesondere die Seiten zu Lehre, Projekte und Einzelseiten der Mitarbeiter.

3) Siehe dazu http://www.hamburger-bildungsser ver.de/startseite/module.pdf oder http://www.unihamburg.de/onTEAM/grafik/1107511876/mate rialband_zum_schlussbericht.pdf. 
4) Siehe dazu Kollek 2004; vgl. auch Feuerstein 2007.

5) $\mathrm{Zu}$ den Projekten s. a. http://www.uni-ham burg.de/fachbereiche-einrichtungen/fg_ta_med/ projekte.html.

6) Vergleiche Kollek, Lemke 2008, S. 179ff.

\section{Literatur}

Beusmann, V., 2007: Dialogische Formen der Politikberatung aus Sicht universitärer Technikfolgenabschätzung, -bewertung und -gestaltung (TA) zur Grünen Gentechnik. In: Cropp, C.; Schiller, F; Wagner, J. (Hg.): Die Zukunft der Wissenskommunikation. Perspektiven für einen reflexiven Dialog von Wissenschaft und Politik - am Beispiel des Agrarbereichs. Berlin, S. 129-149

Deutscher Hochschulverband (Hg.), 2009: Kein Studium mehr ohne Wissenschaftsgeschichte und Ethik. Presseerklärung am 20.3.2009; http://www.his. de/presse/archiv_hn/ganze_pm (download 7.10.09)

Dörpinghaus, A., 2009: Bildung. Plädoyer wider die Verdummung. In: Forschung \& Lehre, Supplement, 9 (2009), S. 1-14

Feuerstein, G., 2007: Folgenspektrum und Bewertung biomedizinischer Techniken. Einführende Vorlesungen. Berlin

Kollek, R., 2004: BioMedical Technology Assessment: Modulare Folgenerfassung und perspektivensensitive Bewertung biomedizinischer Innovationen. In: TATuP 13/3 (2004), S. 85-88

Kollek, R. Lemke, T., 2008: Der medizinische Blick in die Zukunft. Gesellschaftliche Implikationen prädiktiver Gentests. Frankfurt a. M.

Nowotny, H., Testa, G., 2009: Mein Haus, mein Auto, meine DNA. Essay zum Gen-Zeitalter. In: Spiegel Online, 20.10.2009; http://www.spiegel.de/wissen schaft/mensch/0,1518,654621-2,00.html (download 27.10.09)

Peccei, A. (Hg.), 1979: Das menschliche Dilemma. Zukunft und lernen. Club of Rome, Bericht für die achtziger Jahre. Wien, München, Zürich

Silbereisen, R.K., 1997: Das veränderungsoffene und grenzenbewusste Ich - seine Entwicklung über die gesamte Lebensspanne. In: von Weizsäcker, E.U.: Grenzen-los? Jedes System braucht Grenzen - aber wie durchlässig müssen diese sein? Berlin, S. $180-198$

VDI - Verein Deutscher Ingenieure, 1991: Technikbewertung - Begriffe und Grundlagen (Richtlinie 3780). Düsseldorf

Winter, St., 2009: Schickt eure Kinder auf die schönen Umwege! In: Forschung und Lehre 16/9 (2009), S. 633

\section{Kontakt}

Prof. Volker Beusmann

Universität Hamburg

Forschungsschwerpunkt Biotechnik, Gesellschaft und Umwelt (FSP BIOGUM)

Ohnhorststr. 18, 22609 Hamburg

E-Mail: beusmann@uni-hamburg.de

Internet: http://www.biogum.uni-hamburg.de 